EPiC Series in Computing
Volume 80, 2021, Pages 1-31

\title{
ARCH-COMP21 Category Report: Continuous and Hybrid Systems with Linear Continuous Dynamics
}

\author{
Matthias Althoff ${ }^{1}$, Erika Ábrahám ${ }^{2}$, Marcelo Forets ${ }^{3}$, Goran Frehse ${ }^{4}$, Daniel \\ Freire $^{3}$, Christian Schilling ${ }^{5,6}$, Stefan Schupp ${ }^{7}$, and Mark Wetzlinger ${ }^{1}$ \\ 1 Technical University of Munich, Department of Informatics, Munich, Germany \\ althoff@in.tum.de, m.wetzlinger@tum.de \\ 2 RWTH Aachen University, Aachen, Germany \\ abraham@cs.rwth-aachen.de \\ 3 Universidad de la República, Montevideo, Uruguay \\ mforets@gmail.com, dfreire@fisica.edu.uy \\ 4 ENSTA Paris, Palaiseau, France \\ goran.frehse@ensta-paris.fr \\ 5 University of Konstanz, Konstanz, Germany \\ 6 Aalborg University, Aalborg, Denmark \\ christianms@cs.aau.dk \\ 7 TU Wien, Wien, Austria \\ stefan.schupp@tuwien.ac.at
}

\begin{abstract}
We present the results of the $\mathrm{ARCH}^{1} 2021$ friendly competition for formal verification of continuous and hybrid systems with linear continuous dynamics. In its fifth edition, four tools have been applied to solve nine different benchmark problems in the category for linear continuous dynamics (in alphabetical order): CORA, HyDRA, JuliaReach, and SpaceEx. This report is a snapshot of the current landscape of tools and the types of benchmarks they are particularly suited for. Due to the diversity of problems, we are not ranking tools, yet the presented results provide one of the most complete assessments of tools for the safety verification of continuous and hybrid systems with linear continuous dynamics up to this date.
\end{abstract}

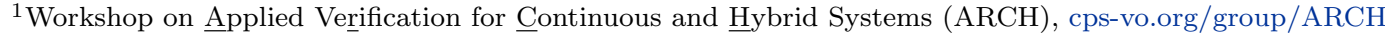




\section{Introduction}

Disclaimer The presented report of the ARCH friendly competition for continuous and hybrid systems with linear continuous dynamics aims at providing a landscape of the current capabilities of verification tools. We would like to stress that each tool has unique strengths - not all of the specificities can be highlighted within a single report. To reach a consensus in what benchmarks are used, some compromises had to be made so that some tools may benefit more from the presented choice than others.

We consider the verification of hybrid systems (i.e., mixed discrete/continuous systems) with linear continuous dynamics

$$
\dot{x}(t)=A x(t)+B u(t),
$$

where $A \in \mathbb{R}^{n \times n}, x \in \mathbb{R}^{n}, B \in \mathbb{R}^{n \times m}$, and $u \in \mathbb{R}^{m}$. For all results reported by each participant, we have run an independent repeatability evaluation. To establish further trustworthiness of the results, the code with which the results have been obtained is publicly available at gitlab.com/goranf/ARCH-COMP. The selection of the benchmarks has been conducted within the forum of the ARCH website (cps-vo.org/group/ARCH), which is visible for registered users and registration is open to anybody. All tools presented in this report use some form of reachability analysis. This, however, is not a constraint set by the organizers of the friendly competition. We hope to encourage further tool developers to showcase their results in future editions. All tools are run on a AWS g4dn.4xlarge machine with 16 Xeon vCPUs and 64 GB RAM. Although we use the same machine, one still has to factor in the efficiency of the programming language of the tools.

A novelty compared to last year is that all models are provided in SpaceEx format on our central repository. By this means, we ensure that all tools use the same model. Once all tools can automatically import SpaceEx models, it will be much easier to handle a larger set of benchmarks.

\section{Participating Tools}

The tools participating in the category Continuous and Hybrid Systems with Linear Continuous Dynamics are subsequently introduced in alphabetical order.

CORA (Matthias Althoff, Mark Wetzlinger) The tool COntinuous Reachability Analyzer (CORA) $[2,6,7,4]$ realizes techniques for reachability analysis with a special focus on developing scalable solutions for verifying hybrid systems with nonlinear continuous dynamics and/or nonlinear differential-algebraic equations. A further focus is on considering uncertain parameters and system inputs. Due to the modular design of CORA, much functionality can be used for other purposes that require resource-efficient representations of multi-dimensional sets and operations on them. CORA is implemented as an object-oriented MATLAB code. CORA is available at cora.in.tum.de. 
HyDRA (Stefan Schupp, Erika Ábrahám) The Hybrid systems Dynamic Reachability Analysis (HyDRA) tool implements flow-pipe construction based reachability analysis for linear hybrid automata. The tool is built on top of HyPro [34] available at ths.rwthaachen.de/research/projects/hypro/, a C++ library for reachability analysis. HyPro provides different implementations of set representations tailored for reachability analysis, such as boxes, convex polyhedra, support functions, or zonotopes, all sharing a common interface. This interface allows one to easily exchange the utilized set representation in HyDRA. We use this to extend state-of-the art reachability analysis by CEGAR-like parameter refinement loops, which (among other parameters) allow us to vary the used set representation.

JuliaReach (Marcelo Forets, Daniel Freire, Christian Schilling) JuliaReach is a software suite for reachability computations of dynamical systems [15], available at http://juliareach.com/. It is written in Julia, a modern high-level language for scientific computing. For the set computations we use the LazySets library [33], which is also part of JuliaReach. JuliaReach can analyze systems in either continuous-time or discrete-time semantics. For some of the models we use our custom $S X$ parser for parsing SX (SpaceEx format) model files, and otherwise we create the models in Julia. Compared to last year we have continued to improve algorithmic aspects in the core component library ReachabilityAnalysis. In this competition we use the following algorithms: BFFPSV18 (based on support functions on low-dimensional subspaces [16]), GLGM06 (based on zonotopes [27]), ASB07 (based on zonotopes for parametric systems [11]), and LGG09 (based on support functions [30]). These algorithms can be combined with different approximation models, such as forward and correction hull, adapted from [22] and [1], respectively. For hybrid systems with time-triggered transitions, we use the algorithm from [20].

SpaceEx (Goran Frehse) SpaceEx is a tool for computing reachability of hybrid systems with complex, high-dimensional dynamics [22, 23, 21]. It can handle hybrid automata whose continuous and jump dynamics are piecewise affine with nondeterministic inputs. Its input language facilitates the construction of complex models from automata components that can be com-

bined to networks and parameterized to construct new components. The analysis engine of SpaceEx combines explicit set representations (polyhedra), implicit set representations (support functions) and linear programming to achieve a maximum of scalability while maintaining high accuracy. It constructs an overapproximation of the reachable states in the form of template polyhedra. Template polyhedra are polyhedra whose faces are oriented according to a user-provided set of directions (template directions). A cover of the continuous trajectories is obtained by time-discretization with an adaptive time-step algorithm. The algorithm ensures that the approximation error in each template direction remains below a given value. SpaceEx is available at http://spaceex.imag.fr.

\section{$3 \quad$ Verification of Benchmarks}

For the 2021 edition, we have decided to keep all benchmarks from our 2020 friendly competition [5] and have added the clamped beam benchmark.

Special Features We briefly list the special features of each benchmark: 
- Heat $3 D$ benchmark from [13]: This is a purely continuous benchmark resulting from a spatial discretization of a heat partial differential equation in three dimensions. The system can be scaled from a $5 \times 5 \times 5$ mesh $(125$ dimensions) to a $100 \times 100 \times 100$ mesh (one million dimensions), each variation being roughly an order of magnitude apart.

- Clamped beam benchmark from [31, Sec. 4.2]: This purely continuous benchmark models a spatially discretized beam clamped on one end and pulled on the other end yielding interesting oscillations. The system dimension ranges from 200 to 2000 depending on the number of nodes used for the discretization. A challenge of this benchmark is that it has very little damping.

- Space station benchmark from [37]: This is a purely continuous benchmark of medium size with 270 state variables and three inputs.

- Spacecraft rendezvous benchmark from [17]: This benchmark has hybrid dynamics and is a linearization of a benchmark in the other ARCH-COMP category Continuous and Hybrid Systems with Nonlinear Dynamics. Consequently, the reader can observe the difference in computation time and verification results between the linearized version and the original dynamics.

- Powertrain benchmark from [8, Sec. 6]: This is a hybrid system for which one can select the number of continuous state variables and the size of the initial set. Up to 51 continuous state variables are considered.

- Building benchmark from [37, No. 2]: A purely continuous linear system with a small number of continuous state variables; the benchmark does not only have safety properties, but also properties that should be violated to check whether the reachable sets contain certain states.

- Platooning benchmark from [14]: A rather small number of continuous state variables is considered, but one can arbitrarily switch between two discrete states: a normal operation mode and a communication-failure mode.

- Gearbox benchmark from [18]: This benchmark has the smallest number of continuous state variables, but the reachable set does not converge to a steady state and the reachable set for one point in time might intersect multiple guards at once.

- Brake benchmark from [36]: This hybrid benchmark has a time-triggered discrete transition that has to be taken 1,001 times.

Types of Inputs Generally, we distinguish between three types of inputs:

1. Fixed inputs, where $u(t)$ is precisely known. In some cases, $u(t)=$ const as in the gearbox benchmark.

2. Uncertain but constant inputs, where $u(t) \in \mathcal{U} \subset \mathbb{R}^{m}$ is uncertain within a set $\mathcal{U}$, but each uncertain input is constant over time: $u(t)=$ const.

3. Uncertain, time-varying inputs $u(t) \in \mathcal{U} \subset \mathbb{R}^{m}$ where $u(t) \neq$ const. Those systems do not converge to a steady state solution and consider uncertain inputs of all frequencies. For tools that cannot consider arbitrarily varying inputs, we have stated that changes in inputs are only considered at fixed points in time. 
Different Paths to Success When tools use a fundamentally different way of solving a benchmark problem, we add further explanations.

Computation Time The computation times specified in this report include the computation time of the reachable set and the time needed for the verification of the specifications.

\subsection{Heat3D}

\subsubsection{Model}

Using a mesh, the Heat3D benchmark is a spatially-discretized partial differential equation (PDE) for heat transfer in three dimensions, resulting in ordinary differential equations (ODEs), where each variable represents a mesh point. Depending on the granularity of the discretization, one can adjust the number of variables. This system has no switching or inputs and serves to evaluate the scalability with respect to the number of system dimensions. It is an academic example, although modifications, such as external inputs or more complicated specifications, can be added in the future. This benchmark was used in [13] and is based on a $2 \mathrm{D}$ version originally described and evaluated in [29, 28].

All of the sides of the considered heated block are insulated, except the $x=1$ edge, causing heat exchange with the ambient environment with a heat exchange constant of 0.5 . A heated initial region is present in the region, where $x \in[0.0,0.4], y \in[0.0,0.2]$, and $z \in[0.0,0.1]$. The entire initial heated region is the same temperature, which is nondeterministic and chosen in the range 0.9 to 1.1 , with the remaining material initially at temperature 0.0 . The system dynamics is given by the heat equation $\operatorname{PDE} u_{t}=\alpha^{2}\left(u_{x x}+u_{y y}+u_{z z}\right)$, where $\alpha=0.01$ is the diffusivity of the material.

A linear model of the system is obtained using the semi-finite difference method, discretizing the block with an $m \times m \times m$ grid. This results in an $m^{3}$-dimensional linear system describing the evolution of the temperature at each mesh point.

Due to the initially heated region, we expect the temperature at the center of the block to first increase, and then decrease due to the heat loss along the $x=1$ edge. Further, the discretization error increases for smaller $m$ motivating the higher-dimensional versions of the benchmark. We suggest a time bound of $T=40$ and a step size of 0.02 (2000 steps).

\subsubsection{Specifications}

The goal is to find the maximum temperature reached at the center of a $1 \times 1 \times 1$ block, where one edge of the block is initially heated. This can be converted to a safety verification problem by checking that $T_{\max }$ is reachable but $T_{\max }+\delta$ is not, for some small $\delta$ like $10^{-4}$.

There are five suggested sizes, roughly each one an order of magnitude apart in terms of the number of dimensions. The higher-dimensional versions usually prevent explicitly representing the dynamics as a dense matrix in memory. Storing a million by million dense matrix requires a trillion numbers, which at 8 bytes per double-precision number would require eight terabytes of storage.

HEAT01 $5 \times 5 \times 5$ (125 dimensions). Note: the initial set is modified to be heated when $z \in[0.0,0.2]$ (single mesh point), since that is the best we can do with this granularity. $T_{\max }: 0.10369$ at time 9.44 .

HEAT02 $10 \times 10 \times 10$ (1000 dimensions). $T_{\max }: 0.02966$ at time 25.5 . 
HEAT03 $20 \times 20 \times 20$ (8000 dimensions). $T_{\max }: 0.01716$ at time 22.62 .

HEAT0 $50 \times 50 \times 50(125,000$ dimensions $) . T_{\max }: 0.01161$ at time 18.88.

HEAT05 $100 \times 100 \times 100(1,000,000$ dimensions $) . T_{\max }: 0.01005$ at time 17.5.

\subsubsection{Results}

Plots for the $5 \times 5 \times 5$ case are shown in Figure 1. Results are shown in Table 1 .

Note CORA CORA applies the reachability algorithm in [24] with a time step size of 0.02 for the benchmark instance HEAT01. For the higher-dimensional benchmark instances we compute the reachable set using the Krylov-subspace-based reachability algorithm in [3] using a time step size of 0.05 for HEAT02 and 0.005 for HEAT03.

Note HyDRA We use a time step size of 0.03 for the instance HEAT01 and a time step size of 0.014 for the instance HEAT02. Both models are verified using a recently added, more efficient implementation of support functions.

Note JuliaReach For HEAT03 and HEAT04 we use an implementation of LGG09 that lazily computes the matrix exponential using the Lanczos algorithm [32], with chosen Krylov subspace dimension of 94 and 211, respectively.

Note SpaceEx SpaceEx computes the matrix exponential with a Padé approximation on dense matrices. It therefore does not scale well to more than $\approx 500$ variables.

Table 1: Computation Times for the Heat3D Benchmark in [s].

\begin{tabular}{|c|c|c|c|c|c|c|}
\hline tool & HEAT01 & HEAT02 & HEAT03 & HEAT04 & HEAT05 & language \\
\hline CORA & 2.2 & 9.3 & 289 & - & - & MATLAB \\
\hline HyDRA & 13.2 & 160 & - & - & - & $\mathrm{C}++$ \\
\hline JuliaReach & 0.13 & 32 & - & - & - & Julia \\
\hline SpaceEx & 4.2 & - & - & - & - & $\mathrm{C}++$ \\
\hline \multicolumn{7}{|c|}{ discrete-time tools } \\
\hline JuliaReach & 0.023 & 1.2 & 137 & 5273 & - & Julia \\
\hline
\end{tabular}

\subsection{Clamped Beam}

\subsubsection{Model}

Similarly to the Heat3D benchmark from above, the Clamped Beam benchmark [31, Sec. 4.2] also results from the spatial discretization of a partial differential equation (PDE), where each variable represents a node along the beam. The number of states scales proportionally with the number of nodes used for the discretization and the system is influenced by a single external input. 


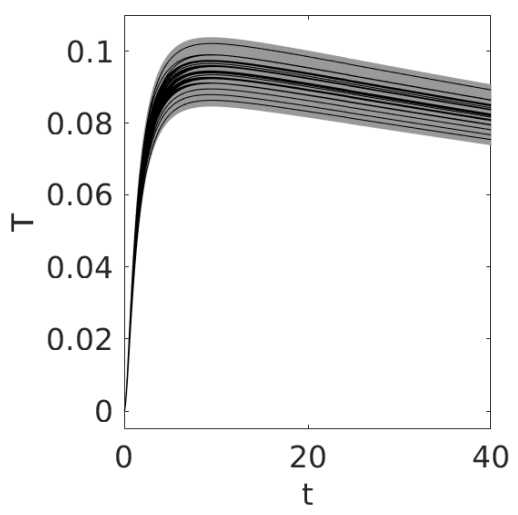

(a) CORA.

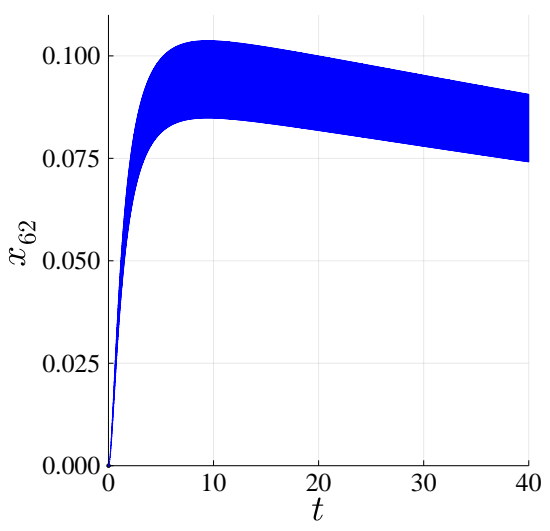

(c) JuliaReach.

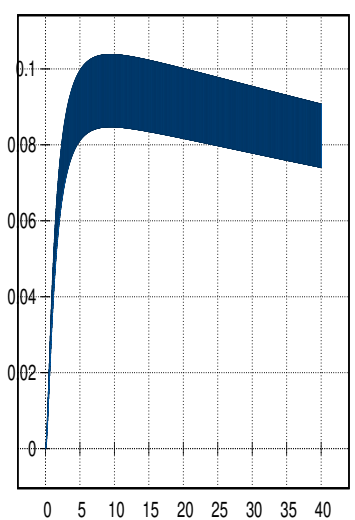

(b) HyDRA.

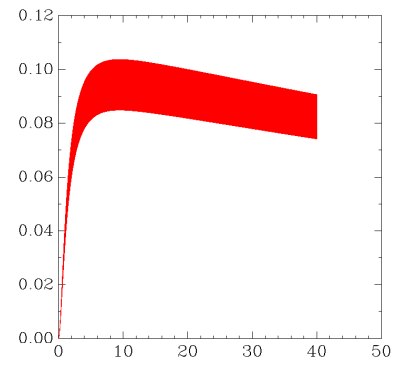

(d) SpaceEx.

Figure 1: Heat3D: Reachable sets for the temperature at the center of the block over time for benchmark version HEAT01. Some tools additionally show possible trajectories.

A beam of length $L$ is fixed at one end, while an external load $F(t)$ acts on the other end. Further model parameters are the cross-section area $A$ as well as the Young modulus $E$ and the density $\rho$ of the material. The governing partial differential equation models the displacement $u(x, t)$ as a function of the position $x$ along the beam and the time $t: E A u_{x x}-\rho A u_{t t}=0$. Please note that the indices indicate partial derivatives with respect to the indexed variables. To obtain a linear system, the beam is spatially discretized using $N$ nodes from $x=0$ to $x=L$ depending on the stiffness matrix $K \in \mathbb{R}^{N \times N}$ and the mass matrix $M \in \mathbb{R}^{N \times N}$. The original model is extended by introducing a damping matrix $D=a K+b M$, where $a=b=10^{-6}$, to model a more realistic beam. Rewriting the equation into a first-order system of linear ODEs yields a system dimension of $2 N$ describing the displacement and velocity of each node over time. The sparsity pattern reveals four blocks of size $N \times N$ : The block $(1,1)$ is all-zero, the block $(1,2)$ is the identity matrix, and the blocks $(2,1)$ and $(2,2)$ are tridiagonal matrices. 
The initial condition is chosen such that all nodes have displacement and velocity zero, i.e., $\forall x \in[0, L]: u(x, 0)=0, u_{t}(x, 0)=0$. The boundary condition keeps the displacement at the fixed end zero at all times so that $\forall t: u(0, t)=0$. The load is modeled by $F(t)=10000 H(t)$ with $H(t)$ denoting the Heaviside function. Finally, the time horizon is set to $T=0.01$. For discrete-time tools, a step size of $9.88 \cdot 10^{-7}$ is used.

\subsubsection{Specifications}

The goal is to find the maximum displacement and maximum velocity reached at the position $x=0.7 L$. For this year, we only have one suggested size, but this is to be extended in the future.

CB01 $N=100$ (200 dimensions).

The load $F(t)$ is modeled in two different ways:

CBC0x (constant inputs) The inputs are uncertain only in their initial value and constant over time: $F(0) \in \mathcal{F}, \dot{F}(t)=0$, with $\mathcal{F}=[9900,10100]$.

CBF0x (time-varying inputs) The inputs can change arbitrarily over time: $\forall t: F(t) \in \mathcal{F}$, with $\mathcal{F}=[9900,10100]$.

Note that the load $F(t)$ of the original model is different from the input $u(t)$ to the spatially discretized system $\dot{x}(t)=A x(t)+u(t)$.

\subsubsection{Results}

Plots for the velocity of the node at $x=0.7 \mathrm{~L}$, corresponding to node 70 are shown in Figure 2 over the time interval $t \in[0,0.01]$. The computation times and the accuracy measure $\max _{t} v_{70}$ are shown in Table 2.

Note CORA For CBC01, the CORA standard algorithm from [24] with a time step size of $1.429 \times 10^{-6}$ (7000 steps) was used, whereas for CBF01, we applied the wrapping-free algorithm from [26] with a time step size of $1.0 \times 10^{-6}$ (10000 steps). In both cases, the zonotope order was tuned to 25 .

Note JuliaReach We use LGG09 and step size $10^{-6}$ and $10^{-7}$ for CBC01 and CBF01, respectively.

Note SpaceEx We use LGG with template direction $x_{170}$ and adaptive time steps. In order to obtain a reachability specification, we checked whether $x_{170} \geq x_{\max }$ is reachable, where $x_{\max }$ was a user-provided value.

\subsection{International Space Station Benchmark}

\subsubsection{Model}

The International Space Station (ISS) is a continuous linear time-invariant system $\dot{x}(t)=$ $A x(t)+B u(t)$ proposed as a benchmark in ARCH 2016 [37]. In particular, the considered system is a structural model of the component 1R (Russian service module), which has 270 state variables with three inputs. 


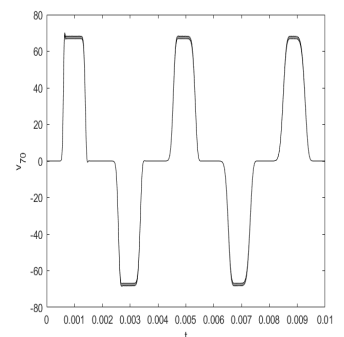

(a) CORA.

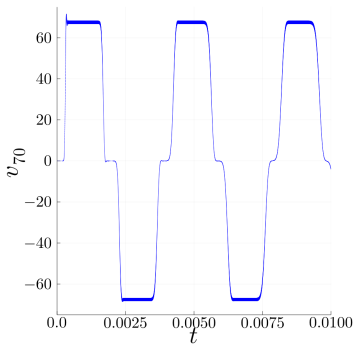

(b) JuliaReach.

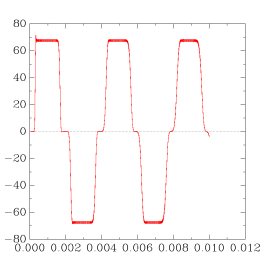

(c) SpaceEx.

Figure 2: Clamped Beam CBC01: Reachable sets for the velocity at node 70. Some tools additionally show possible trajectories.

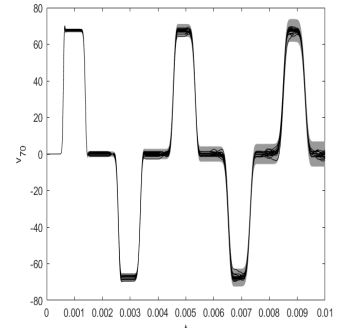

(a) CORA.

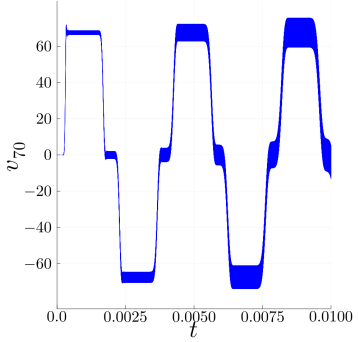

(b) JuliaReach.

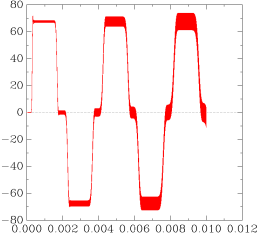

(c) SpaceEx.

Figure 3: Clamped Beam CBF01: Reachable sets for the velocity at node 70. Some tools additionally show possible trajectories.

Initially, all 270 variables are in the range $[-0.0001,0.0001], u_{1}$ is in $[0,0.1], u_{2}$ is in $[0.8,1]$, and $u_{3}$ is in $[0.9,1]$. The time bound is 20 . Discrete-time analysis for the space station benchmark should be done with a step size of 0.01 . The A, B, and C matrices are available in MATLAB format ${ }^{2}$ (that can also be opened with Python using scipy.io.loadmat) and in SpaceEx format ${ }^{3}$. There are two versions of this benchmark:

Table 2: Computation Times in $[\mathrm{s}]$ and accuracy measure $\max _{t} v_{70}$ for the Clamped Beam benchmark.

\begin{tabular}{lccccc}
\hline tool & \multicolumn{2}{c}{ CBC01 } & \multicolumn{2}{c}{ CBF01 } & language \\
& Time & $\max _{t} v_{70}$ & Time & $\max _{t} v_{70}$ & \\
\hline CORA & 7.1 & 70.11 & 30 & 73.78 & MATLAB \\
JuliaReach & 1.4 & 71.63 & 12 & 75.63 & Julia \\
SpaceEx & 312.78 & 71.7 & 318.88 & 74 & $\mathrm{C}++$ \\
\hline \multicolumn{5}{c}{ discrete-time tools } \\
JuliaReach & 0.6 & 71.6 & 0.5 & 73.78 & Julia \\
\hline
\end{tabular}

\footnotetext{
${ }^{2}$ slicot.org/objects/software/shared/bench-data/iss.zip

${ }^{3}$ cps-vo.org/node/34059
} 
ISSF01 The inputs can change arbitrarily over time: $\forall t: u(t) \in \mathcal{U}$.

ISSC01 (constant inputs) The inputs are uncertain only in their initial value and constant over time: $u(0) \in \mathcal{U}, \dot{u}(t)=0$.

\subsubsection{Specifications}

The verification goal is to check the ranges reachable by the output $y_{3}$, which is a linear combination of the state variables $\left(y=C x, C \in \mathbb{R}^{3 \times 270}\right)$. In addition to the safety specification, for each version there is an UNSAT instance that serves as a sanity check to ensure that the model and the tool work as intended. But there is a caveat: In principle, verifying an UNSAT instance only makes sense formally if a witness is provided (counter-example, underapproximation, etc.). Since most of the participating tools do not have this capability, we run the tools with the same accuracy settings on an SAT-UNSAT pair of instances. The SAT instance demonstrates that the over-approximation is not too coarse, and the UNSAT instance demonstrates that the over-approximation is indeed conservative, at least in the narrow sense of the specification.

ISS01 Bounded time, safe property: For all $t \in[0,20], y_{3}(t) \in[-0.0007,0.0007]$. This property is used with the uncertain input case (ISSF01) and assumed to be satisfied.

ISS02 Bounded time, safe property: For all $t \in[0,20], y_{3}(t) \in[-0.0005,0.0005]$. This property is used with the constant input case (ISSC01) and assumed to be satisfied.

ISU01 Bounded time, unsafe property: For all $t \in[0,20], y_{3}(t) \in[-0.0005,0.0005]$. This property is used with the uncertain input case (ISSF01) and assumed to be unsatisfied.

ISU02 Bounded time, unsafe property: For all $t \in[0,20], y_{3}(t) \in[-0.00017,0.00017]$. This property is used with the constant input case (ISSC01) and assumed to be unsatisfied.

\subsubsection{Results}

Results of the international space station benchmark for state $y_{3}$ over time are shown in Fig. 4 and Fig. 5. The computation times of various tools for the benchmark are listed in Tab. 3.

Note CORA CORA applies the block-decomposition algorithm [16] with step size 0.01 and zonotope order 30 for benchmark version ISSF01. For version ISSC01, a step size of 0.02 and a zonotope order of 10 is used.

Note JuliaReach We use the LGG09 implementation. The step sizes in dense time are $6 \times 10^{-4}$ for ISSF01 and $1 \times 10^{-2}$ for ISSC01.

Note SpaceEx SpaceEx was run with the LGG algorithm. The sampling was chosen as 0.005 for ISSF01 and 0.05 for ISSC01. Only the two template directions $\pm y_{3}$ were used. Since $y_{3}$ is an algebraic variable that is a linear expression of the state variables, we replaced it in the forbidden states and the direction definition by the corresponding linear expression. To model the constant inputs in ISSC01, we introduced $u_{1}, u_{2}, u_{3}$ as state variables with $\dot{u}_{1}=\dot{u}_{2}=\dot{u}_{3}=$ 0. A custom algorithm for constant inputs could avoid such an artificial augmentation and 
significantly reduce the runtime for ISSC01. Note that SpaceEx treats the initial states as a general polyhedron, i.e., a linear program is solved at every time step. SpaceEx also computes the full matrix exponential, a $270 \times 270$ matrix, even though in the LGG algorithm it would suffice to compute the vector $e^{A t} \ell$ for each template direction $\ell$.

Since SpaceEx does not currently support the plotting of algebraic variables, we used the following trick to plot $y_{3}$ over time: we introduced a state variable $z$ with dynamics $\dot{z}=$ $-1000\left(z-y_{3}\right)$. Since the time constant for $z$ is about two orders of magnitude below that of $y_{3}$, we expect the plots to be practically identical to a true plot of $y_{3}$.

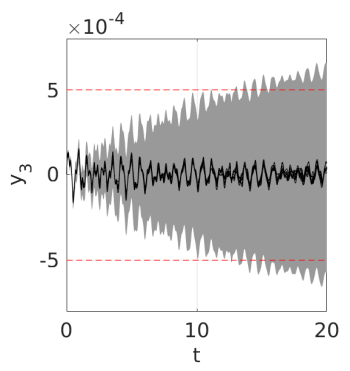

(a) CORA.

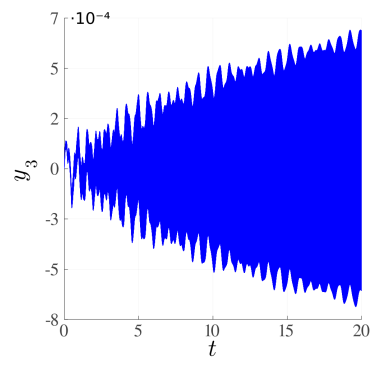

(b) JuliaReach.

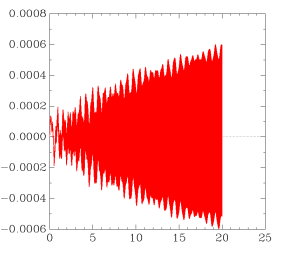

(c) SpaceEx.

Figure 4: ISS: Reachable sets of $y_{3}$ plotted over time for the uncertain input case.

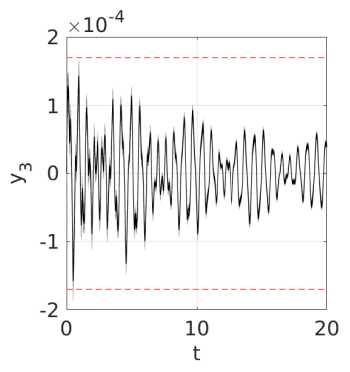

(a) CORA.

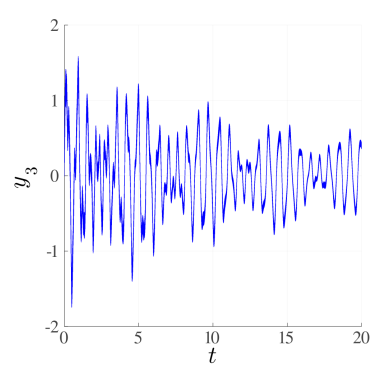

(b) JuliaReach.

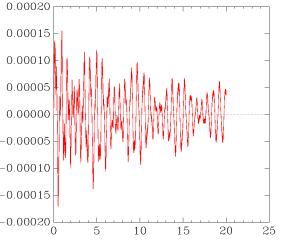

(c) SpaceEx

Figure 5: ISS: Reachable sets of $y_{3}$ plotted over time for the constant input case.

\subsection{Spacecraft Rendezvous Benchmark}

\subsubsection{Model}

Spacecraft rendezvous is a perfect use case for formal verification of hybrid systems since mission failure can cost lives and is extremely expensive. This benchmark is taken from [17]; its original continuous dynamics is nonlinear, and the original system is verified in the ARCHCOMP category Continuous and Hybrid Systems with Nonlinear Dynamics. When spacecraft are in close proximity (such as rendezvous operations), a common approximation to analyze the nonlinear dynamics is to use the linearized Clohessy-Wiltshire-Hill (CWH) equations [19]. This benchmark analyzes this linear hybrid model. 
Table 3: Computation Times for the International Space Station Benchmark in [s].

\begin{tabular}{lcccccc}
\hline & \multicolumn{2}{c}{ ISSF01 } & & \multicolumn{2}{c}{ ISSC01 } & \\
\cline { 2 - 3 } tool & ISS01 & ISU01 & & ISS02 & ISU02 & language \\
\hline CORA & 59 & 38 & & 1.3 & 0.072 & MATLAB \\
JuliaReach & 10 & 10 & & 1.4 & 1.4 & Julia \\
SpaceEx & 49 & 48 & 29 & 29 & C ++ \\
\hline \multicolumn{5}{c}{ discrete-time tools } \\
JuliaReach & 10 & 10 & 1.4 & 1.4 & Julia \\
\hline
\end{tabular}

The hybrid nature of this benchmark originates from a switched controller, while the dynamics of the spacecraft is purely continuous. In particular, the modes are approaching $(100 \mathrm{~m}-$ $1000 \mathrm{~m}$ ), rendezvous attempt (less than $100 \mathrm{~m}$ ), and aborting. Discrete-time analysis for the rendezvous system should be done with a step size of 0.1 . The model is available in C2E2, SDVTool, and SpaceEx format on the ARCH website ${ }^{4}$. The set of initial states is

$$
\mathcal{X}_{0}=\left[\begin{array}{c}
-900 \\
-400 \\
0 \\
0
\end{array}\right] \oplus\left[\begin{array}{c}
{[-25,25]} \\
{[-25,25]} \\
0 \\
0
\end{array}\right] \text {. }
$$

The following benchmark instances are considered:

SRNA01 The spacecraft approaches the target as planned and there exists no transition into the aborting mode.

SRA01 A transition into aborting mode occurs at time $t=120$ [min].

SRA02 A transition into aborting mode occurs nondeterministically, $t \in[120,125]$ [min].

SRA03 A transition into aborting mode occurs nondeterministically, $t \in[120,145]$ [min].

SRA04 A transition into aborting mode occurs at time $t=240$ [min].

SRA05 A transition into aborting mode occurs nondeterministically, $t \in[235,240]$ [min].

SRA06 A transition into aborting mode occurs nondeterministically, $t \in[230,240]$ [min].

SRA07 A transition into aborting mode occurs nondeterministically, $t \in[50,150]$ [min].

SRA08 A transition into aborting mode occurs nondeterministically, $t \in[0,240]$ [min].

An initial, discrete-time analysis indicated it is safe to enter the aborting mode up to around time $t=250$ [min]. We also added the following two instances, which are presumably unsafe. For timing, tools should use the same settings for these as for the safe cases.

SRU01 A transition into aborting mode occurs at time $t=260$ [min].

SRU02 A transition into aborting mode occurs nondeterministically, $t \in[0,260]$ [min].

\footnotetext{
${ }^{4}$ cps-vo.org/node/36349
} 


\subsubsection{Specifications}

Given the thrust constraints of the specified model, in mode rendezvous attempt, the absolute velocity must stay below $0.055 \mathrm{~m} / \mathrm{s}$. In the aborting mode, the vehicle must avoid the target, which is modeled as a box $\mathcal{B}$ with $0.2 \mathrm{~m}$ edge length and the center placed as the origin. In the rendezvous attempt the spacecraft must remain within the line-of-sight cone $\mathcal{L}=\left\{[x, y]^{T} \mid(x \geq\right.$ $\left.-100 m) \wedge\left(y \geq x \tan \left(30^{\circ}\right)\right) \wedge\left(-y \geq x \tan \left(30^{\circ}\right)\right)\right\}$. It is sufficient to check these parameters for a time horizon of 300 minutes.

Let us denote the discrete state by $z(t)$ and the continuous state vector by $x(t)=$ $\left[s_{x}, s_{y}, v_{x}, v_{y}\right]^{T}$, where $s_{x}$ and $s_{y}$ are the positions in $\mathrm{x}$ - and y-direction, respectively, and $v_{x}$ and $v_{y}$ are the velocities in $\mathrm{x}$ - and $\mathrm{y}$-direction, respectively. The mode approaching is denoted by $z_{1}$, the mode rendezvous attempt by $z_{2}$, and the mode aborting by $z_{3}$. We can formalize the specification as

$$
\begin{gathered}
\text { SR02 } \forall t \in[0,300 \text { min }], \forall x(0) \in \mathcal{X}_{0}:\left(z(t)=z_{2}\right) \Longrightarrow\left(\sqrt{v_{x}^{2}+v_{y}^{2}} \leq 0.055 \mathrm{~m} / \mathrm{s} \wedge\right. \\
\left.\left[s_{x}, s_{y}\right]^{T} \in \mathcal{L}\right) \wedge\left(z(t)=z_{3}\right) \Longrightarrow\left(\left[s_{x}, s_{y}\right]^{T} \notin \mathcal{B}\right) .
\end{gathered}
$$

To solve the above specification, all tools under-approximate the nonlinear constraint $\sqrt{v_{x}^{2}+v_{y}^{2}} \leq 0.055 \mathrm{~m} / \mathrm{s}$ by an octagon as shown in Fig. 6 .

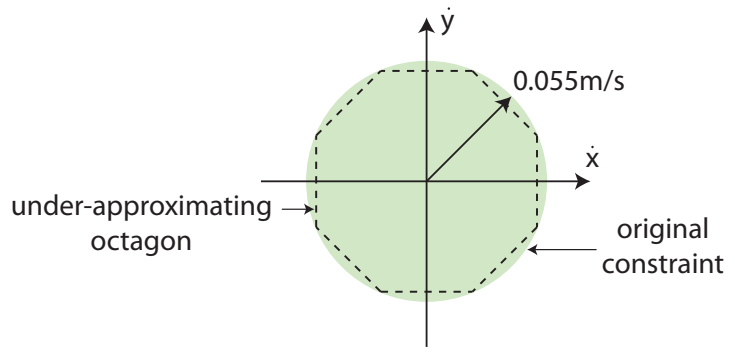

Figure 6: Under-approximation of the nonlinear velocity constraint by an octagon.

Remark on nonlinear constraint In the original benchmark, the constraint on the velocity was set to $0.05 \mathrm{~m} / \mathrm{s}$, but it can be shown that this constraint cannot be satisfied by a counterexample. For this reason, we have relaxed the constraint to $0.055 \mathrm{~m} / \mathrm{s}$.

\subsubsection{Results}

Results of the spacecraft rendezvous benchmark for the $s_{x}$ - $s_{y}$-plane are shown for the version SRNA01 in Fig. 7 and for the version SRA01 in Fig. 8. The computation times of various tools for the spacecraft rendezvous benchmark are listed in Tab. 4.

Note CORA For both benchmark versions, CORA was run with a zonotope order of 10 and with the following step sizes: $0.2[\mathrm{~min}]$ for the mode approaching, 0.02 [min] for the mode rendezvous attempt, and 0.2 [min] for the mode aborting (does not exist for version SRNA01). Intersections with deterministic guards are calculated with the method of Girard and Le Guernic in [25]. In order to find suitable orthogonal directions for the method in [25], we perform the following procedure: first, we project the last zonotope not intersecting the guard set onto the 
guard set; second, we apply principal component analysis to the generators of the projected zonotope, providing us with the orthogonal directions. For non-deterministic guards we first unite all reachable sets intersecting the guard set and then compute the intersection using constrained zonotopes [35].

Note JuliaReach We use BFFPSV18 and chose a one-block partition and hyperrectangular reach sets with a step size in dense time of 0.04 for instances SRA01-SRA03. We handle discrete transitions by computing the intersection with invariants and guards lazily before their overapproximation with a hyperrectangle. For the instance SRA04, we use a clustering strategy of order 40 and step size of 0.01 . For the unsat instances we use the step size 0.04 .

Note SpaceEx SpaceEx was run with the LGG algorithm, box directions, and a flowpipe tolerance of 0.2 .

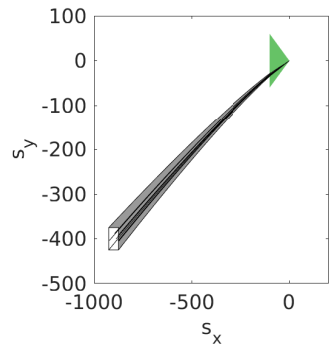

(a) CORA.

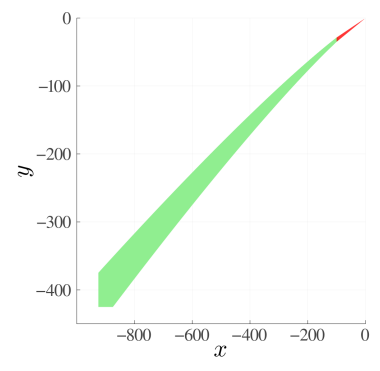

(b) JuliaReach.

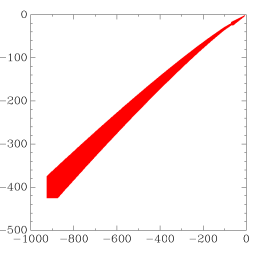

(c) SpaceEx.

Figure 7: Reachable sets for the spacecraft rendezvous benchmark in the $s_{x}-s_{y}$-plane for the benchmark variant without maneuver abortion (SRNA01).

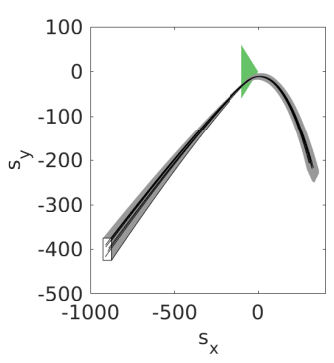

(a) CORA.

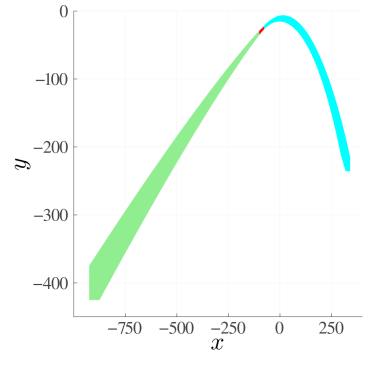

(b) JuliaReach.

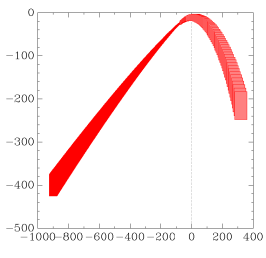

(c) SpaceEx.

Figure 8: Reachable sets for the spacecraft rendezvous benchmark in the $s_{x}-s_{y}$-plane for the benchmark variant with maneuver abortion at $t=120$ [min] (SRA01, over analysis time horizon of 300 [min]) 
Table 4: Computation time [s] for the spacecraft rendezvous benchmarks $\left(\mathbf{S R}^{*}\right)$ for specification SR02.

\begin{tabular}{|c|c|c|c|c|c|c|c|c|c|c|c|}
\hline tool & NA01 & A01 & A02 & A03 & A04 & A05 & A06 & A07 & A08 & U01 & U02 \\
\hline CORA & 13 & 2.6 & 3.5 & 5.4 & 12 & 16 & 16 & 31 & 90 & 12 & 95 \\
\hline JuliaReach & 0.34 & 0.35 & 0.37 & 0.57 & 36 & - & - & - & - & 3.7 & 7.7 \\
\hline SpaceEx & 0.21 & 0.22 & - & - & - & - & - & - & - & 4.2 & 21 \\
\hline \multicolumn{12}{|c|}{ discrete-time tools } \\
\hline JuliaReach & 0.12 & 0.14 & 0.14 & 0.24 & - & - & - & - & - & - & 4.3 \\
\hline
\end{tabular}

\subsection{Powertrain with Backlash}

\subsubsection{Model}

The powertrain benchmark is an extensible benchmark for hybrid systems with linear continuous dynamics taken from $[8$, Sec. 6$]$ and $[12$, Sec. 4]. The essence of this benchmark is recalled here, and the reader is referred to the above-cited papers for more details. The benchmark considers the powertrain of a vehicle consisting of its motor and several rotating masses representing different components of the powertrain, e.g., gears, differential, and clutch, as illustrated in Fig. 9. The benchmark is extensible in the sense that the number of continuous states can be easily extended to $n=7+2 \theta$, where $\theta$ is the number of additional rotating masses. The number of discrete modes, however, is fixed and originates from backlash, which is caused by a physical gap between two components that are normally touching, such as gears. When the rotating components switch direction, for a short time they temporarily disconnect, and the system is said to be in the dead zone. The model is available in SpaceEx format on the ARCH website ${ }^{5}$. The set of initial states is

$$
\begin{aligned}
\mathcal{X}_{0} & =\{c+\alpha g \mid \alpha \in[-1,1]\}, \\
c & =[-0.0432,-11,0,30,0,30,360,-0.0013,30, \ldots,-0.0013,30]^{T}, \\
g & =[0.0056,4.67,0,10,0,10,120,0.0006,10, \ldots, 0.0006,10]^{T} .
\end{aligned}
$$

\subsubsection{Specifications}

We analyze an extreme maneuver from a maximum negative acceleration that lasts for $0.2[\mathrm{~s}]$, followed by a maximum positive acceleration that lasts for 1.8 [s]. The initial states of the model are on a line segment in the $n$-dimensional space. We create different difficulty levels of the reachability problem by scaling down the initial states by some percentage. The model has the following non-formal specification: after the change of direction of acceleration, the powertrain completely passes the dead zone before being able to transmit torque again. Due to oscillations in the torque transmission, the powertrain should not re-enter the dead zone of the backlash.

To formalize the specification using linear time logic (LTL), let us introduce the following discrete states:

- $z_{1}$ : left contact zone

\footnotetext{
${ }^{5}$ cps-vo.org/node/49115
} 


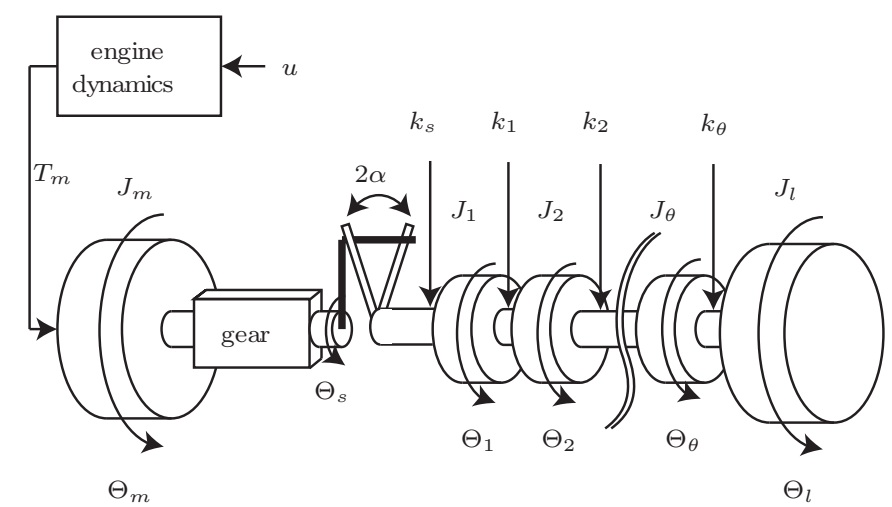

Figure 9: Powertrain model.

- $z_{2}$ : dead zone

- $z_{3}$ : right contact zone

For all instances, the common specification is: For all $t \in[0,2], x(0) \in \mathcal{X}_{0},\left(z_{2} U z_{3}\right) \Longrightarrow$ $G\left(z_{3}\right)$. The instances only differ in the size of the system and the initial set, where center $(\cdot)$ returns the volumetric center of a set.

DTN01 $\theta=2, \mathcal{X}_{0}:=0.05\left(\mathcal{X}_{0}-\operatorname{center}\left(\mathcal{X}_{0}\right)\right)+\operatorname{center}\left(\mathcal{X}_{0}\right)$.

DTN02 $\theta=2, \mathcal{X}_{0}:=0.3\left(\mathcal{X}_{0}-\operatorname{center}\left(\mathcal{X}_{0}\right)\right)+\operatorname{center}\left(\mathcal{X}_{0}\right)$.

DTN03 $\theta=2$, no change of $\mathcal{X}_{0}$.

DTN04 $\theta=22, \mathcal{X}_{0}:=0.05\left(\mathcal{X}_{0}-\operatorname{center}\left(\mathcal{X}_{0}\right)\right)+\operatorname{center}\left(\mathcal{X}_{0}\right)$.

DTN05 $\theta=22, \mathcal{X}_{0}:=0.3\left(\mathcal{X}_{0}-\operatorname{center}\left(\mathcal{X}_{0}\right)\right)+\operatorname{center}\left(\mathcal{X}_{0}\right)$.

DTN06 $\theta=22$, no change of $\mathcal{X}_{0}$.

\subsubsection{Results}

Results of the powertrain benchmark in the $x_{1}-x_{3}$-plane are shown in Fig. 10. The computation times of various tools for the powertrain benchmark are listed in Tab. 5.

Note CORA CORA uses the following time step sizes: $0.005 s$ for DTN01, DTN02, and DTN03; $0.002 s$ for DTN04; and $0.001 s$ for DTN05 and DTN06. For all benchmark versions, CORA was run with a zonotope order of 20 . The intersections with the guard sets are calculated with the approach from [25], and principal component analysis is used to find suitable directions for the enclosure of the guard intersections.

Note JuliaReach We use the GLGM06 algorithm with a step size of 0.001. In addition we use slightly different analysis parameters for different modes. 


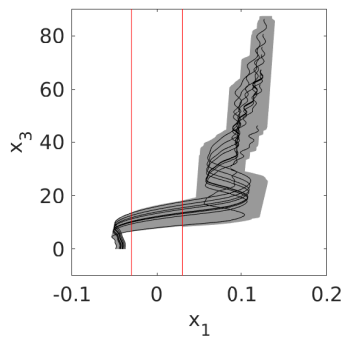

(a) CORA (DTN03).

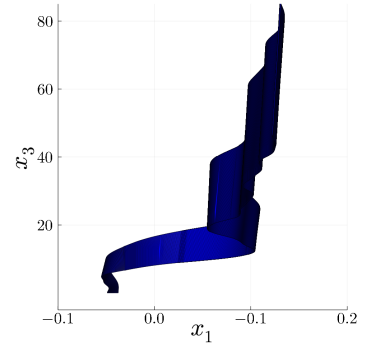

(b) JuliaReach (DTN03).

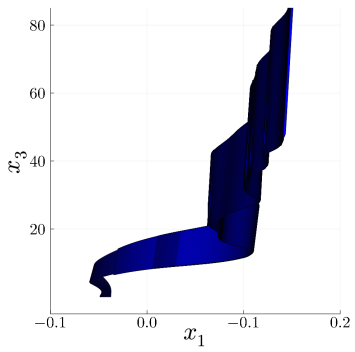

(c) JuliaReach (DTN05)

Figure 10: Reachable sets in the $x_{1}-x_{3}$-plane.

Table 5: Computation Times for the Powertrain Benchmark in [s].

\begin{tabular}{lccccccc}
\hline tool & DTN01 & DTN02 & DTN03 & DTN04 & DTN05 & DTN06 & language \\
\hline CORA & 5.6 & 5.4 & 5.5 & 39 & 74 & 212 & MATLAB \\
JuliaReach & 0.2 & 0.2 & 0.1 & 0.3 & 0.7 & 0.8 & Julia \\
\hline
\end{tabular}

\subsection{Building Benchmark}

\subsubsection{Model}

This benchmark is quite straightforward: The system is described by $\dot{x}(t)=A x(t)+B u(t)$, $u(t) \in \mathcal{U}, y(t)=C x(t)$, where $A, B, C$ are provided on the ARCH website ${ }^{6}$. The initial set and the uncertain input $\mathcal{U}$ are provided in [37, Tab. 2.2]. Discrete-time analysis for the building system should use a step size of 0.01 . There are two versions of this benchmark:

BLDF01 The inputs can change arbitrarily over time: $\forall t: u(t) \in \mathcal{U}$.

BLDC01 (constant inputs) The inputs are uncertain only in their initial value, and constant over time: $u(0) \in \mathcal{U}, \dot{u}(t)=0$. The purpose of this model instance is to accommodate tools that cannot handle time-varying inputs.

\subsubsection{Specifications}

The verification goal is to check whether the displacement $y_{1}$ of the top floor of the building remains below a given bound. In addition to the safety specification from the original benchmark, there are two UNSAT instances that serve as sanity checks to ensure that the model and the tool work as intended. But there is a caveat: In principle, verifying an UNSAT instance only makes sense formally if a witness is provided (counter-example, under-approximation, etc.). Since most of the participating tools do not have this capability, we run the tools with the same accuracy settings on an SAT-UNSAT pair of instances. The SAT instance demonstrates that the over-approximation is not too coarse, and the UNSAT instance indicates that the over-approximation is indeed conservative.

BDS01 Bounded time, safe property: For all $t \in[0,20], y_{1}(t) \leq 5.1 \cdot 10^{-3}$. This property is assumed to be satisfied.

\footnotetext{
${ }^{6}$ cps-vo.org/node/34059
} 
BDU01 Bounded time, unsafe property: For all $t \in[0,20], y_{1}(t) \leq 4 \cdot 10^{-3}$. This property is assumed to be violated. Property BDU01 serves as a sanity check. A tool should be run with the same accuracy settings on BLDF01-BDS01 and BLDF01-BDU01, returning UNSAT on the former and SAT on the latter.

BDU02 Bounded time, unsafe property: The forbidden states are $\left\{y_{1}(t) \leq-0.78 \cdot 10^{-3} \wedge t=20\right\}$. This property is assumed to be violated for BLDF01 and satisfied for BLDC01. Property BDU02 serves as a sanity check to confirm that time-varying inputs are taken into account. A tool should be run with the same accuracy settings on BLDF01-BDU02 and BLDC01BDU02, returning UNSAT on the former and SAT on the latter.

\subsubsection{Results}

Results of the building benchmark for state $x_{25}$ over time are shown in Fig. 11-14. The computation times of various tools for the building benchmark are listed in Tab. 6 .

Note CORA Since the dynamics of this example is dominated by the input after one second, we use the step size 0.002 for $t \in[0,1]$ and the step size 0.01 for $t \in[1,20]$. The zonotope order is chosen as 100 .

Note HyDRA We use a step size of 0.004 and support functions with an octagonal template as a state set representation. As HyDRA cannot handle uncertain inputs, we have added another variable to the model accounting for the uncertain input.

Note JuliaReach Since the safety property only involves one state variable, we use the LGG09 algorithm. Here we use the step sizes (in dense time) 0.004 for BLDF01 and 0.006 for BLDC01.

Note SpaceEx The accuracy of SpaceEx was set to the largest value possible that satisfies the specification, here $\varepsilon=0.01$. This means the tool can exploit any margin to reduce the number of computations and/or the number of convex sets in the reach set. The resulting, intentional lack of accuracy shows in the plot.

Table 6: Computation Times for the Building Benchmark in [s].

\begin{tabular}{lccc}
\hline tool & $\begin{array}{c}\text { BLDC01 } \\
\text { BDS01 }\end{array}$ & $\begin{array}{c}\text { BLDF01 } \\
\text { BDS01 }\end{array}$ & language \\
\hline CORA & 2.9 & 3.3 & MATLAB \\
HyDRA & 0.426 & - & $\mathrm{C}++$ \\
JuliaReach & 0.0096 & 0.012 & Julia \\
SpaceEx & 1.6 & 1.8 & $\mathrm{C}++$ \\
\hline & discrete-time tools & \\
JuliaReach & 0.0021 & 0.0025 & Julia \\
\hline
\end{tabular}




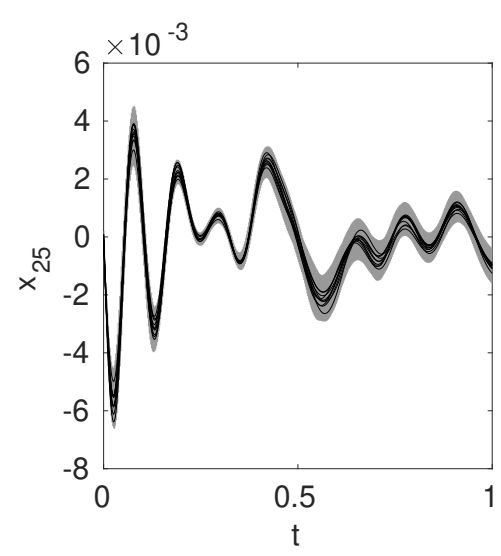

(a) CORA.

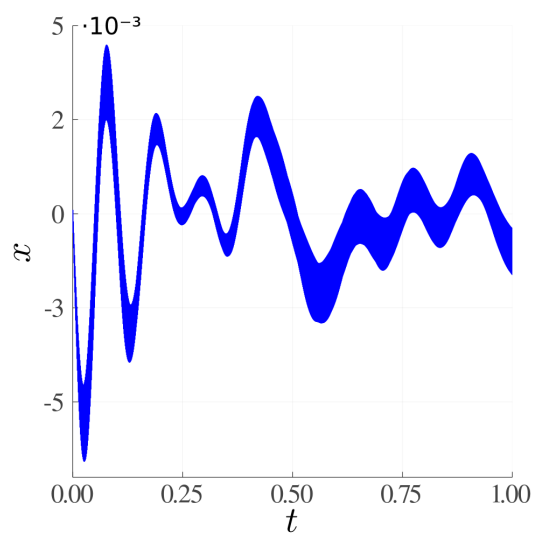

(b) JuliaReach.

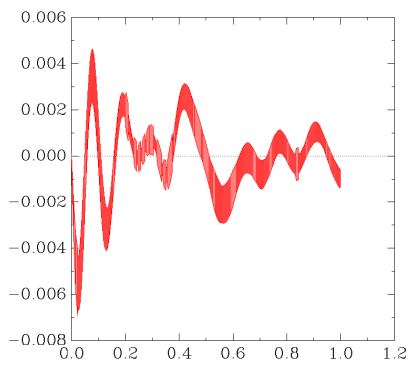

(c) SpaceEx.

Figure 11: Building (BLDF01): Reachable sets of $x_{25}$ plotted over time up to time 1. Some tools additionally show possible trajectories.

\subsection{Platooning Benchmark}

\subsubsection{Model}

The platooning benchmark considers a platoon of three vehicles following each other. This benchmark considers loss of communication between vehicles. The initial discrete state is $q_{c}$. Three scenarios are considered for the loss of communication:

PLAA01 (arbitrary loss) The loss of communication can occur at any time, see Fig. 15(a). This includes the possibility of no communication at all.

PLADxy (loss at deterministic times) The loss of communication occurs at fixed points in time, which are determined by clock constraints $c_{1}$ and $c_{2}$ in Fig. 15(b). The clock $t$ is reset when communication is lost and when it is re-established. Note that the transitions have must-semantics, i.e., they take place as soon as possible.

PLAD01: $c_{1}=c_{2}=5$. 


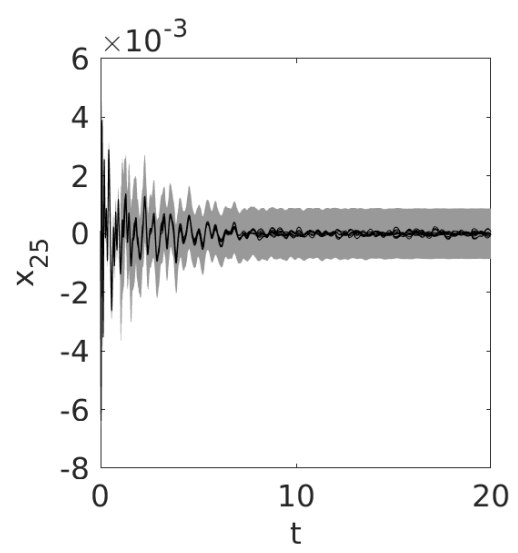

(a) CORA.

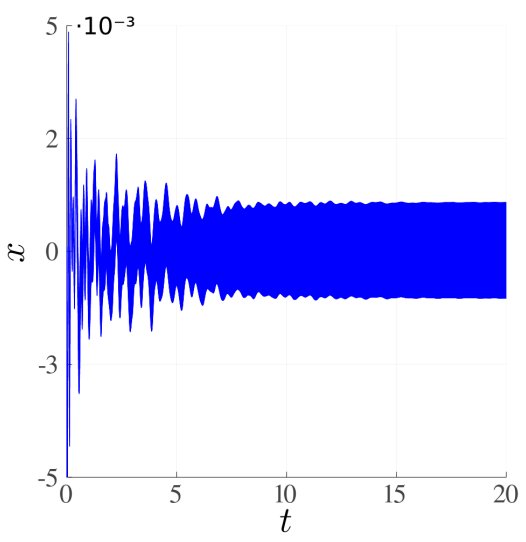

(b) JuliaReach.

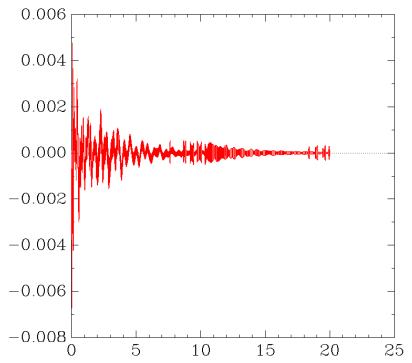

(c) SpaceEx.

Figure 12: Building (BLDF01): Reachable sets of $x_{25}$ plotted over time up to time 20. Some tools additionally show possible trajectories.

PLANxy (loss at nondeterministic times) The loss of communication occurs at any time $t \in\left[t_{b}, t_{c}\right]$ in Fig. 15(c). The clock $t$ is reset when communication is lost and when it is re-established. Communication is reestablished at any time $t \in\left[0, t_{r}\right]$. This scenario covers loss of communication after an arbitrarily long time $t \geq t_{c}$ by reestablishing communication in zero time.

PLAN01: $t_{b}=10, t_{c}=20, t_{r}=20$.

The models are available in SpaceEx, KeYmaera, and MATLAB/Simulink format on the ARCH website $^{7}$. Discrete-time analysis for the platoon system should use a step size of 0.1.

Discussion The arbitrary-loss scenario (PLAA) subsumes the other two instances (PLAD, PLAN).

${ }^{7}$ cps-vo.org/node/15096 


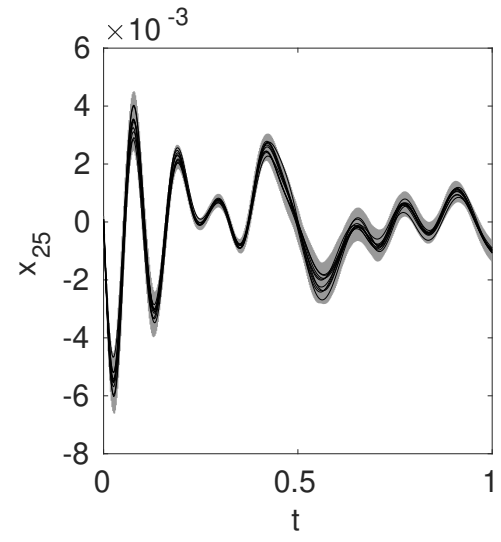

(a) CORA.

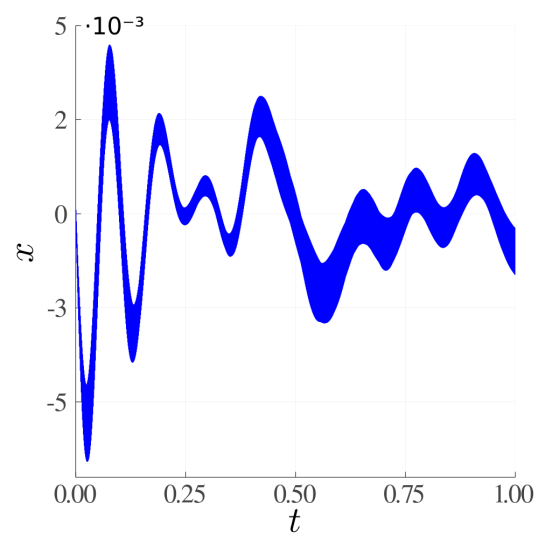

(c) JuliaReach.

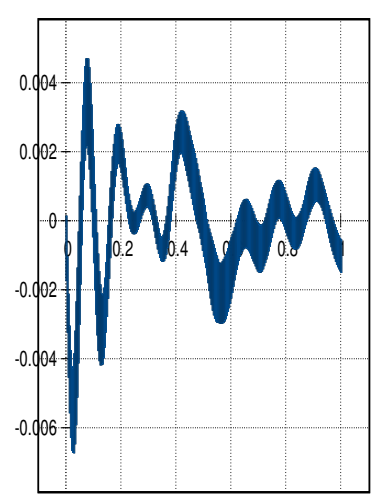

(b) HyDRA.

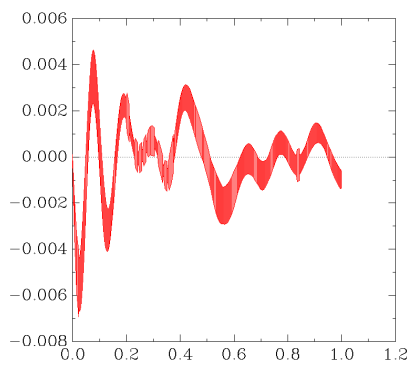

(d) SpaceEx.

Figure 13: Building (BLDC01): Reachable sets of $x_{25}$ plotted over time up to time 1. Some tools additionally show possible trajectories.

\subsubsection{Specifications}

The verification goal is to check whether the minimum distance between vehicles is preserved. The choice of the coordinate system is such that the minimum distance is a negative value.

BNDxy Bounded time (no explicit bound on the number of transitions): For all $t \in[0,20][\mathrm{s}]$, $x_{1}(t) \geq-d_{\min }[\mathrm{m}], x_{4}(t) \geq-d_{\min }[\mathrm{m}]$, and $x_{7}(t) \geq-d_{\min }[\mathrm{m}]$.

BND50: $d_{\min }=50$.

BND42: $d_{\min }=42$.

BND30: $d_{\min }=30$.

UNBxy Unbounded time and unbounded switching: For all $t \geq 0[\mathrm{~s}], x_{1}(t) \geq-d_{\min }[\mathrm{m}]$, $x_{4}(t) \geq-d_{\min }[\mathrm{m}]$, and $x_{7}(t) \geq-d_{\min }[\mathrm{m}]$.

UNB50: $d_{\min }=50$. 


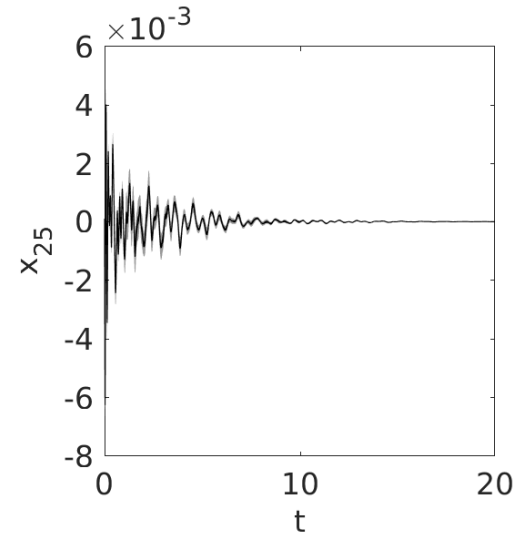

(a) CORA.

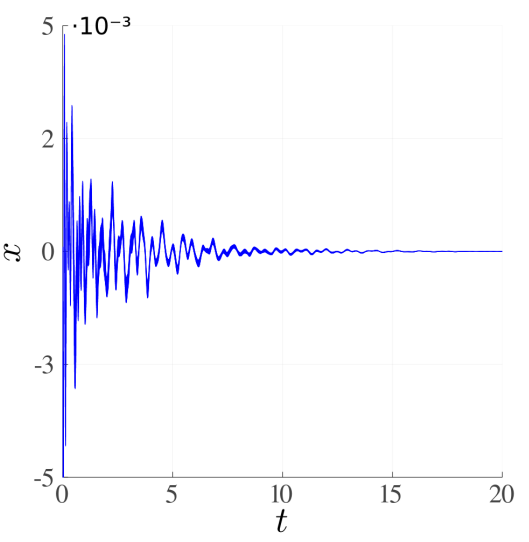

(c) JuliaReach.

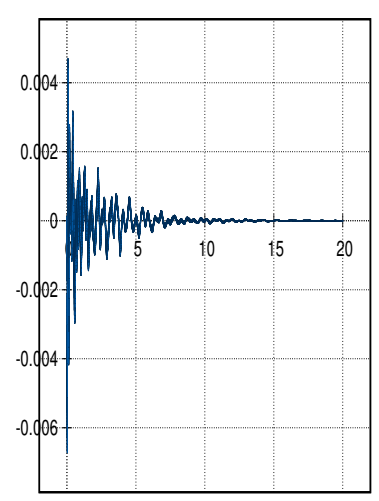

(b) HyDRA.

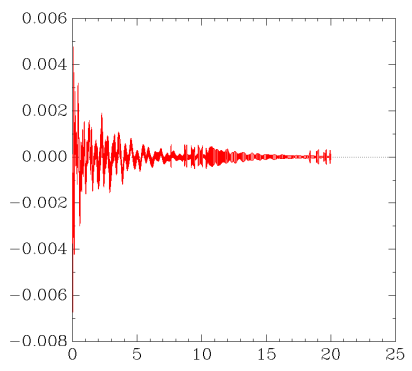

(d) SpaceEx.

Figure 14: Building (BLDC01): Reachable sets of $x_{25}$ plotted over time up to time 20. Some tools additionally show possible trajectories.

$\mathrm{UNB} 42: d_{\min }=42$.

UNB30: $d_{\min }=30$.

\subsubsection{Results}

Results of the platoon benchmark for state $x_{1}$ over time are shown in Fig. 16-18. The computation times of various tools for the platoon benchmark are listed in Tab. 7.

Note CORA CORA was run with the following settings:

- PLAA01-BND50: zonotope order 400 and time step size 0.02 .

- PLAA01-BND42: zonotope order 800 and time step size 0.009 .

- PLAD01-BND42: zonotope order 20 and time step size 0.02s. 


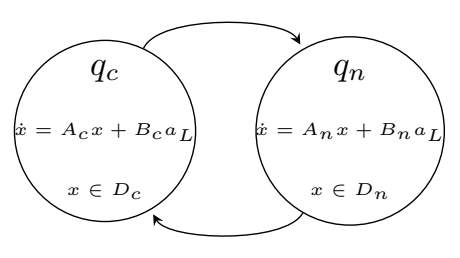

(a) Arbitrary switching.

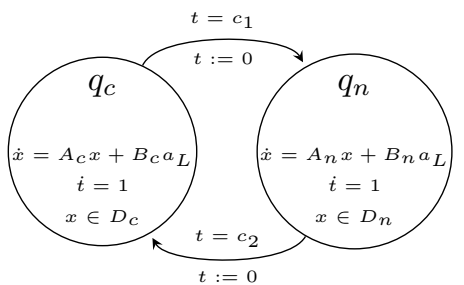

(b) Deterministic switching.

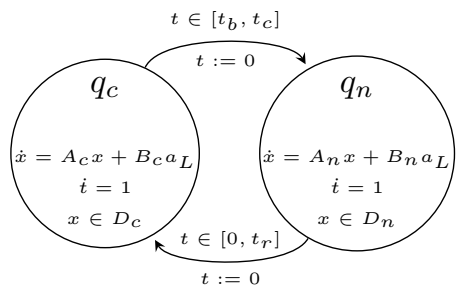

(c) Nondeterministic switching.

Figure 15: Three options adapted from the original benchmark proposal [14]. On the left, the system can switch arbitrarily between the modes. In the middle, mode switches are only possible at given points in time. On the right, mode switches are only possible during given time intervals.

- PLAD01-BND30: zonotope order 200 and time step size 0.02 .

- PLAN01-UNB50: zonotope order 400 and time step size 0.01 . To verify the specification for all times, the reachable set was increased by $1 \%$ at $t=50$ and it was checked whether this set is re-entered.

- PLAA01: we used continuization $[9,10]$ to rewrite the hybrid automaton as a purely continuous system with uncertain parameters.

Note HyDRA As HyDRA cannot handle uncertain time-varying inputs, we use an instance of the platoon benchmark in which inputs are constant (similar to the building benchmark). We use a step size of 0.25 and support functions with an octagonal template for instance DBND30 and a box-shaped template for instance DBND42.

Note JuliaReach For PLAD01-BND42, we use the BFFPSV18 algorithm with a one-block partition, hyperrectangular reach-sets, and a step size (in dense time) of 0.01. For PLAD01BND30, we use the LGG09 algorithm with a step size (in dense time) of 0.03, and intersections with the guard are taken lazily with an octagonal template.

Table 7: Computation Times for the Platoon Benchmark in [s].

\begin{tabular}{|c|c|c|c|c|c|c|}
\hline tool & $\begin{array}{c}\text { PLAA01 } \\
\text { BND50 }\end{array}$ & $\begin{array}{c}\text { PLAA01 } \\
\text { BND42 }\end{array}$ & $\begin{array}{c}\text { PLAD01 } \\
\text { BND42 }\end{array}$ & $\begin{array}{c}\text { PLAD01 } \\
\text { BND30 }\end{array}$ & $\begin{array}{c}\text { PLAN01 } \\
\text { UNB50 }\end{array}$ & language \\
\hline CORA & 13 & 40 & 1.4 & 3.3 & 158 & MATLAB \\
\hline HyDRA & - & - & 1.830 & 104 & - & $\mathrm{C}++$ \\
\hline JuliaReach & - & - & 0.031 & 30 & - & Julia \\
\hline SpaceEx & - & - & 0.36 & 9.8 & 109 & $\mathrm{C}++$ \\
\hline \multicolumn{7}{|c|}{ discrete-time tools } \\
\hline JuliaReach & h $\quad-$ & - & 0.48 & 10 & - & Julia \\
\hline
\end{tabular}




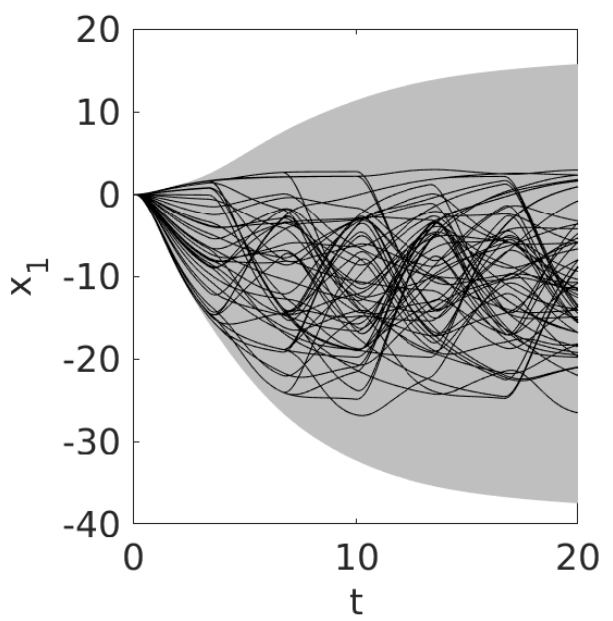

(a) CORA.

Figure 16: PLAA01: Reachable sets of $x_{1}$ plotted over time. CORA additionally shows possible trajectories.

\subsection{Gearbox Benchmark}

\subsubsection{Model}

The gearbox benchmark models the motion of two meshing gears. When the gears collide, an elastic impact takes place. As soon as the gears are close enough, the gear is considered meshed. The model includes a monitor state that checks whether the gears are meshed or free and is available in SpaceEx format ${ }^{8}$ and as a Simulink model ${ }^{9}$. Once the monitor reaches the state meshed, it stays there indefinitely.

With four continuous state variables, the gearbox benchmark has a relatively low number of continuous state variables. The challenging aspect of this benchmark is that the solution heavily depends on the initial state as already pointed out in [18]. For some initial continuous states, the target region is reached without any discrete transition, while for other initial states, several discrete transitions are required.

In the original benchmark, the position uncertainty in the direction of the velocity vector of the gear teeth (x-direction) is across the full width of the gear spline. Uncertainties of the position and velocity in y-direction, which is perpendicular to the x-direction, are considered to be smaller. Due to the sensitivity with respect to the initial set, we consider smaller initial sets. The full uncertainty in $\mathrm{x}$-direction could be considered by splitting the uncertainty in $\mathrm{x}$-direction and aggregating the individual results. For discrete-time analysis of the gearbox system, a step size of 0.0001 (1.0E-4) should be used.

GRBX01: The initial set is $\mathcal{X}_{0}=0 \times 0 \times[-0.0168,-0.0166] \times[0.0029,0.0031] \times 0$.

GRBX02: The initial set is $\mathcal{X}_{0}=0 \times 0 \times[-0.01675,-0.01665] \times[0.00285,0.00315] \times 0$.

\footnotetext{
${ }^{8}$ cps-vo.org/node/34375

${ }^{9}$ cps-vo.org/node/34374
} 


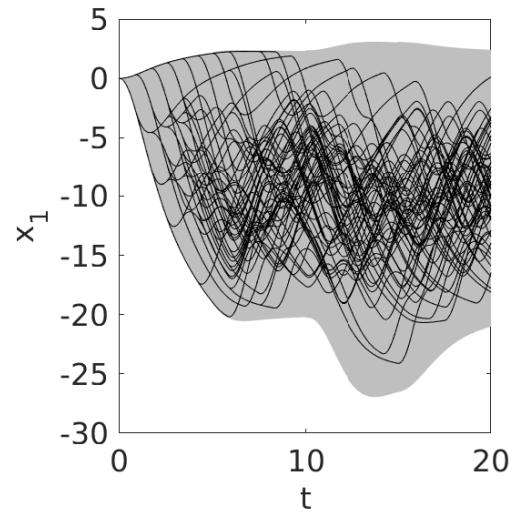

(a) CORA (BND30).

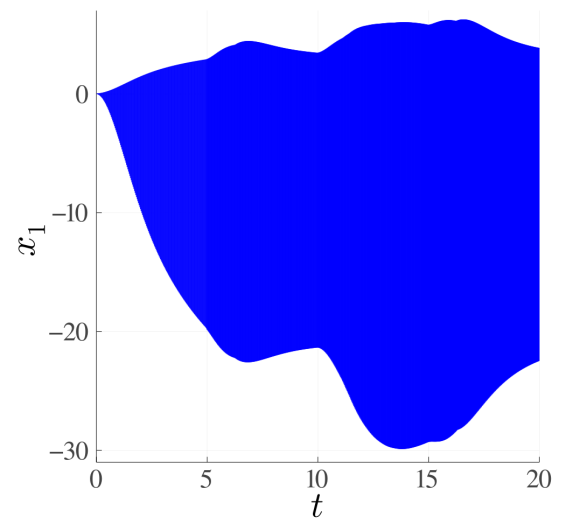

(c) JuliaReach (BND30).

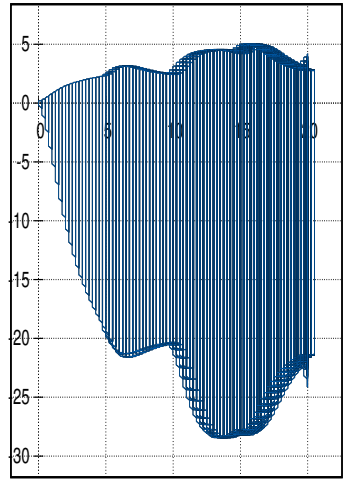

(b) HyDRA (BND30).

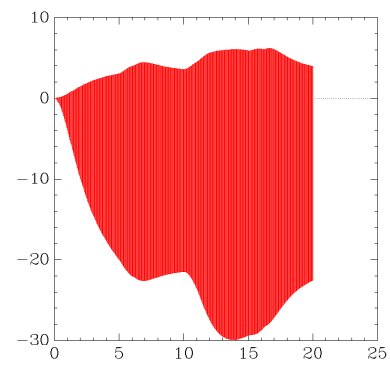

(d) SpaceEx (BND30).

Figure 17: PLAD01: Reachable sets of $x_{1}$ plotted over time. Some tools additionally show possible trajectories.

\subsubsection{Specification}

The goal is to show that the gears are meshed within a time frame of $0.2[\mathrm{~s}]$ and that the bound $x_{5} \leq 20[\mathrm{Nm}]$ of the cumulated impulse is met. Using the monitor states free and meshed, and a global clock $t$, this can be expressed as a safety property as follows: For all $t \geq 0.2$, the monitor should be in meshed. Under nonblocking assumptions, this means that $t<0.2$ whenever the monitor is not in meshed, i.e., when it is in free.

MES01: forbidden states: $($ free $\wedge t \geq 0.2) \vee\left(x_{5} \geq 20\right)$

\subsubsection{Results}

Results of the benchmark for state $x_{3}$ and $x_{4}$ are shown in Fig. 19. The computation times of various tools for the benchmark are listed in Tab. 8. 


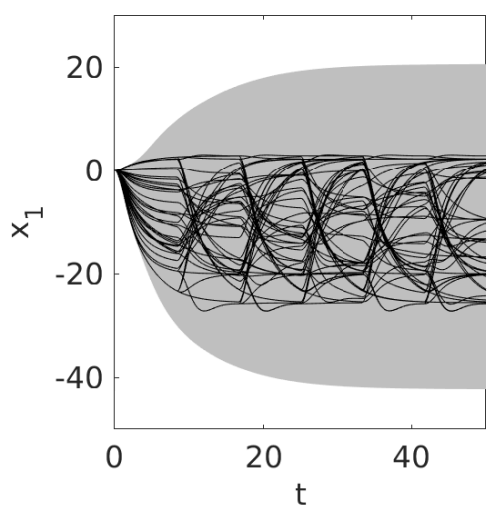

(a) CORA (UNB50).

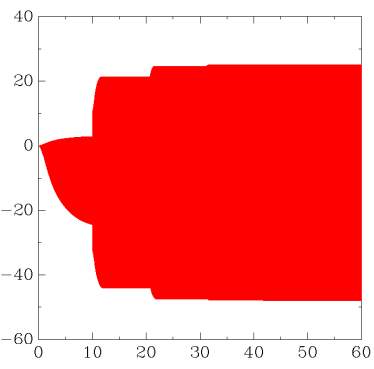

(b) SpaceEx (UNB50).

Figure 18: PLAN01: Reachable sets of $x_{1}$ plotted over time.

Note CORA CORA was run with a time step size of 0.0011 and a zonotope order of 20 . The intersections with the guard sets were calculated with the method of Girard and Le Guernic [25]. In order to find suitable orthogonal directions for the method in [25], we perform the following procedure: first, we project the last zonotope not intersecting the guard set onto the guard set; second, we apply principal component analysis to the generators of the projected zonotope, providing us with the orthogonal directions.

Note JuliaReach We use the LGG09 algorithm with step sizes (in dense time) of 0.0005 (GRBX01) resp. 0.0008 (GRBX02).

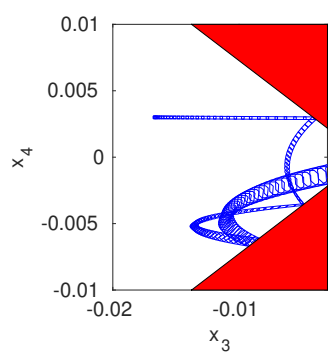

(a) CORA.

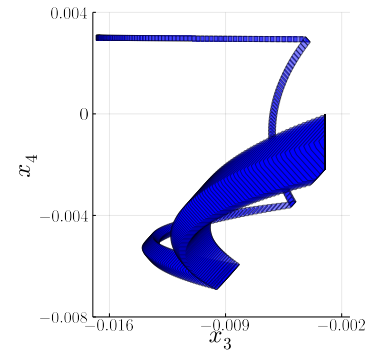

(b) JuliaReach.

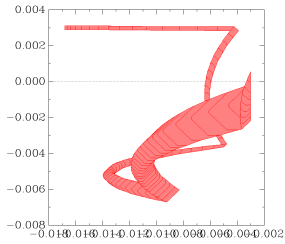

(c) SpaceEx.

Figure 19: Gearbox (GRBX01): Reachable sets of $x_{3}$ and $x_{4}$. 
Table 8: Computation Times of the Gearbox Benchmark in [s].

\begin{tabular}{lccc}
\hline tool & GRBX01-MES01 & GRBX02-MES01 & language \\
\hline CORA & 0.95 & 0.88 & MATLAB \\
JuliaReach & 2.4 & 1.5 & Julia \\
SpaceEx & 0.069 & 0.071 & $\mathrm{C}++$ \\
\hline \multirow{3}{*}{ JuliaReach } & discrete-time tools & & \\
\hline
\end{tabular}

\subsection{Brake Benchmark}

\subsubsection{Model}

The brake benchmark models an electro-mechanical braking system, where a motor pushes a brake caliper against a brake disk that is connected to a (car) wheel [36]. The model describes a closed-loop system comprising a plant model as well as a controller and is representative for challenges in automotive systems. The original Simulink model has been simplified for usage in various analysis tools ${ }^{10}$. Here, we consider a linearized version with parameters.

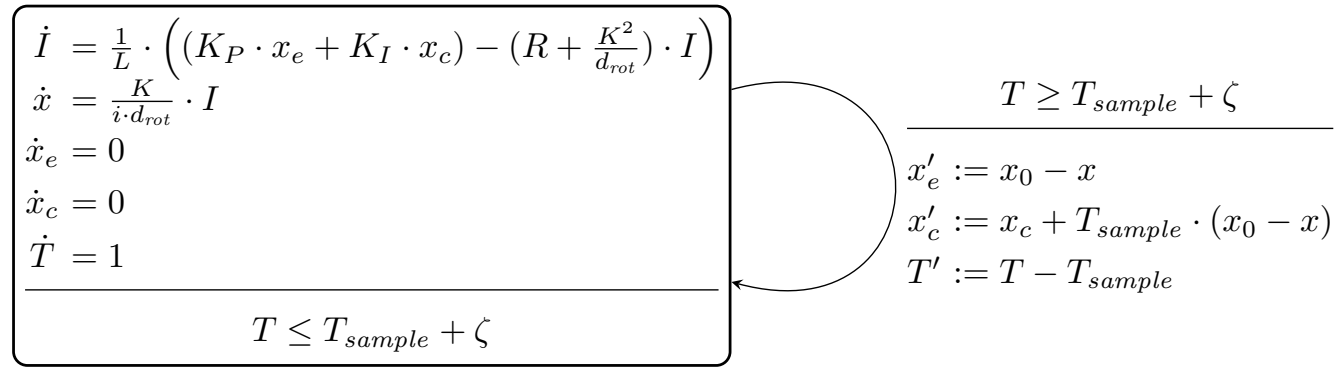

Figure 20: Hybrid automaton of the electro-mechanical brake with periodic discrete-time PI controller and sampling jitter.

The model is a hybrid automaton (see Fig. 20) with four state variables (the motor current $I$, the brake position $x$, and two auxiliary linearization variables) and a clock variable $T$. The automaton consists of a single mode and a self-loop transition. The transition is time-triggered, i.e., it only depends on the value of the clock variable.

We consider two types of uncertainties in the model. The first uncertainty is a variation in the model parameters. We use the settings from [36] for the nonparametric and parametric scenarios. The second uncertainty is sampling jitter (i.e., nondeterministic switching). Unlike the linear model in [36], we consider jitter with a periodic clock (instead of a drifting clock).

\footnotetext{
${ }^{10}$ cps-vo.org/node/20289
} 


\subsubsection{Specification}

While structurally simple, the benchmark is challenging due to the large number of 1,001 discrete jumps within the time horizon 0.1. The initial state is the origin, we use the parameters $x_{0}=0.05$ and $T_{\text {sample }}=10^{-4}$, and in the case of nondeterministic switching the transitions are taken at multiples of $T_{\text {sample }}$ with a nondeterministic jitter from the interval $\zeta=\left[-10^{-8}, 10^{-7}\right]$. We study the property $x<x_{0}$ in both scenarios without and with parameter ranges:

BRKDC01: Verify that $x<x_{0}$ holds for the whole time horizon 0.1 (non-parametric scenario with deterministic switching).

BRKNC01: Same as BRKDC01, but with non-deterministic switching.

BRKNP01: Report the largest time horizon for which $x<x_{0}$ holds (parametric scenario with non-deterministic switching).

\subsubsection{Results}

Results of the benchmark are shown in Fig. 21. The computation times of various tools for the benchmark are listed in Tab. 9.

Note CORA CORA was run with a time step size of $2^{-6}$, a zonotope order of 20 , and the intersections with the non-deterministic guard sets were calculated with constrained zonotopes [35].

Note JuliaReach For the BRKDC01 and BRKNC01 scenario, we use the GLGM06 algorithm with a fixed step size of $10^{-7}$ resp. $10^{-8}$. For the BRKNP01 scenario, we use the ASB07 algorithm with a step size of $10^{-8}$. In all scenarios we use a maximum zonotope order of one. The discrete-time instances use the same step sizes as the dense-time ones. We use a custom analysis for dealing with the time-triggered transition efficiently, considering intersections with the guard separately from the flowpipe computation, as described in [20]. The largest time horizon for which $x<x_{0}$ holds for BRKNP01 is $0.0823 \mathrm{~s}$ and $0.0824 \mathrm{~s}$ for dense and continuous time, respectively.

Note SpaceEx We use the STC algorithm, which here is significantly faster than the LGG algorithm despite using sophisticated algorithms for containment checking, convexification, and redundancy reduction of polyhedra. Since SpaceEx is a model checker, it checks after each jump whether the successor states have already been visited. In this benchmark, all states are in the same location. At the $k$-th jump, this leads to a pairwise containment check with all $k-1$ previous states. This consumes about $90 \%$ of the runtime. For the non-deterministic instance BRKNC01, the required precision means that polyhedra are much more complex than in the deterministic instance (more faces). The containment checking therefore leads to an excessive runtime.

\section{Conclusion and Outlook}

This report presents the results of the fourth friendly competition for the formal verification of continuous and hybrid systems with linear continuous dynamics as part of the ARCH'21 workshop. The reports of other categories can be found in the proceedings and on the ARCH website: cps-vo.org/group/ARCH. 


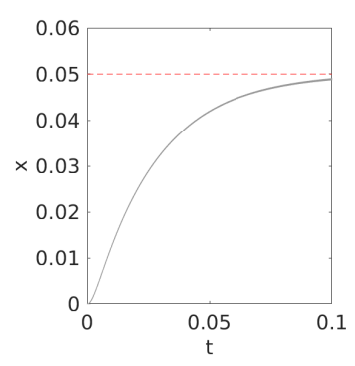

(a) CORA.

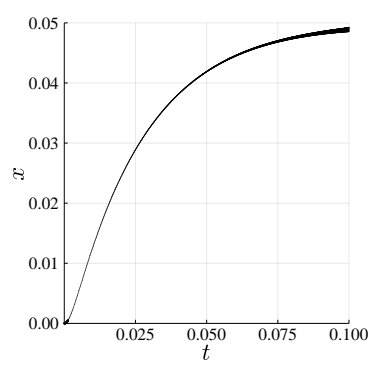

(b) JuliaReach.

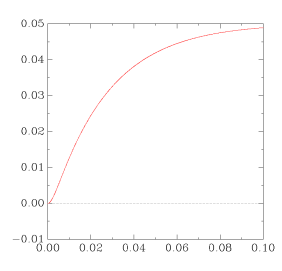

(c) SpaceEx.

Figure 21: Brake: Reachable sets for $x$ over time. Some tools additionally show possible trajectories.

Table 9: Computation Times of the Brake Benchmark in [s].

\begin{tabular}{|c|c|c|c|c|}
\hline tool & BRKDC01 & BRKNC01 & BRKP01 & language \\
\hline CORA & 12 & 603 & 739 & MATLAB \\
\hline JuliaReach & 0.82 & 1.7 & 18 & Julia \\
\hline SpaceEx & 21 & - & - & $\mathrm{C}++$ \\
\hline \multicolumn{5}{|c|}{ discrete-time tools } \\
\hline JuliaReach & 0.081 & 0.56 & 17 & Julia \\
\hline
\end{tabular}

A major observation of the results is that participating tools have significantly reduced computation times compared to the previous year. Also, one can now execute the Dockerfile of all tools on gitlab.com/goranf/ARCH-COMP using the command measure_all. Information about the competition in 2022 will be announced on the ARCH website.

\section{Acknowledgments}

The authors gratefully acknowledge financial support by the European Commission project justITSELF under grant number 817629 and the German Research Foundation project CONVEY under grant number GRK 2428.

\section{References}

[1] M. Althoff. Reachability Analysis and its Application to the Safety Assessment of Autonomous Cars. Dissertation, Technische Universität München, 2010. http://nbnresolving.de/urn/resolver.pl?urn:nbn:de:bvb:91-diss-20100715-963752-1-4.

[2] M. Althoff. An introduction to CORA 2015. In Proc. of the Workshop on Applied Verification for Continuous and Hybrid Systems, page 120-151, 2015.

[3] M. Althoff. Reachability analysis of large linear systems with uncertain inputs in the Krylov subspace. IEEE Transactions on Automatic Control, 65(2):477-492, 2020. 
[4] M. Althoff. Guaranteed state estimation in cora 2021. In Proc. of the 8th International Workshop on Applied Verification for Continuous and Hybrid Systems, 2021.

[5] M. Althoff, S. Bak, M. Forets, G. Frehse, N. Kochdumper, R. Ray, C. Schilling, and S. Schupp. ARCH-COMP19 category report: Continuous and hybrid systems with linear continuous dynamics. In Goran Frehse and Matthias Althoff, editors, Proc. of the 6th International Workshop on Applied Verification of Continuous and Hybrid Systems, volume 61 of EPiC Series in Computing, page 14-40, 2019.

[6] M. Althoff and D. Grebenyuk. Implementation of interval arithmetic in CORA 2016. In Proc. of the 3rd International Workshop on Applied Verification for Continuous and Hybrid Systems, page 91-105, 2016.

[7] M. Althoff, D. Grebenyuk, and N. Kochdumper. Implementation of Taylor models in CORA 2018. In Proc. of the 5th International Workshop on Applied Verification for Continuous and Hybrid Systems, page 145-173, 2018.

[8] M. Althoff and B. H. Krogh. Avoiding geometric intersection operations in reachability analysis of hybrid systems. In Hybrid Systems: Computation and Control, page 45-54, 2012.

[9] M. Althoff, C. Le Guernic, and B. H. Krogh. Reachable set computation for uncertain time-varying linear systems. In Hybrid Systems: Computation and Control, page 93-102, 2011.

[10] M. Althoff, A. Rajhans, B. H. Krogh, S. Yaldiz, X. Li, and L. Pileggi. Formal verification of phase-locked loops using reachability analysis and continuization. Communications of the ACM, 56(10):97-104, 2013.

[11] M. Althoff, O. Stursberg, and M. Buss. Reachability analysis of linear systems with uncertain parameters and inputs. In Proc. of the 46th IEEE Conference on Decision and Control, page 726-732, 2007.

[12] S. Bak, S. Bogomolov, and M. Althoff. Time-triggered conversion of guards for reachability analysis of hybrid automata. In Proc. of the 15th International Conference on Formal Modelling and Analysis of Timed Systems, page 133-150, 2017.

[13] Stanley Bak, Hoang-Dung Tran, and Taylor T Johnson. Numerical verification of affine systems with up to a billion dimensions. In Proceedings of the 22nd ACM International Conference on Hybrid Systems: Computation and Control, pages 23-32, 2019.

[14] I. Ben Makhlouf and S. Kowalewski. Networked cooperative platoon of vehicles for testing methods and verification tools. In Proc. of ARCH14-15. 1st and 2nd International Workshop on Applied veRification for Continuous and Hybrid Systems, page 37-42, 2015.

[15] S. Bogomolov, M. Forets, G. Frehse, K. Potomkin, and C. Schilling. JuliaReach: a toolbox for set-based reachability. In $H S C C, 2019$.

[16] S. Bogomolov, M. Forets, G. Frehse, F. Viry, A. Podelski, and C. Schilling. Reach set approximation through decomposition with low-dimensional sets and high-dimensional matrices. In $H S C C$, pages 41-50, 2018.

[17] N. Chan and S. Mitra. Verifying safety of an autonomous spacecraft rendezvous mission. In Proc. of the 4th International Workshop on Applied Verification of Continuous and Hybrid Systems, page 20-32, 2017.

[18] H. Chen, S. Mitra, and G. Tian. Motor-transmission drive system: a benchmark example for safety verification. In Proc. of ARCH14-15. 1st and 2nd International Workshop on Applied veRification for Continuous and Hybrid Systems, page 9-18, 2015.

[19] WH Clohessy. Terminal guidance system for satellite rendezvous. Journal of the Aerospace Sciences, 27(9):653-658, 1960.

[20] Marcelo Forets, Daniel Freire, and Christian Schilling. Efficient reachability analysis of parametric linear hybrid systems with time-triggered transitions. In MEMOCODE, pages 1-6. IEEE, 2020.

[21] G. Frehse. Reachability of hybrid systems in space-time. In Alain Girault and Nan Guan, editors, Proc. Int. Conf. Embedded Software, EMSOFT, Amsterdam, Netherlands, October 4-9, 2015, pages 41-50. IEEE, 2015. 
[22] G. Frehse, C. Le Guernic, A. Donzé, S. Cotton, R. Ray, O. Lebeltel, R. Ripado, A. Girard, T. Dang, and O. Maler. SpaceEx: Scalable verification of hybrid systems. In Proc. of the 23rd International Conference on Computer Aided Verification, LNCS 6806, page 379-395. Springer, 2011.

[23] G. Frehse, R. Kateja, and C. Le Guernic. Flowpipe approximation and clustering in space-time. In C. Belta and F. Ivancic, editors, Proc. Int. Conf. Hybrid systems: computation and control, HSCC, April 8-11, 2013, Philadelphia, PA, USA, pages 203-212. ACM, 2013.

[24] A. Girard. Reachability of uncertain linear systems using zonotopes. In Hybrid Systems: Computation and Control, LNCS 3414, page 291-305. Springer, 2005.

[25] A. Girard and C. Le Guernic. Zonotope/hyperplane intersection for hybrid systems reachability analysis. In Proc. of Hybrid Systems: Computation and Control, LNCS 4981, page 215-228. Springer, 2008.

[26] A. Girard, C. Le Guernic, and O. Maler. Efficient computation of reachable sets of linear timeinvariant systems with inputs. In Hybrid Systems: Computation and Control, LNCS 3927, page 257-271. Springer, 2006.

[27] Antoine Girard, Colas Le Guernic, and Oded Maler. Efficient computation of reachable sets of linear time-invariant systems with inputs. In HSCC, volume 3927 of LNCS, pages 257-271. Springer, 2006.

[28] Zhi Han. Formal verification of hybrid systems using model order reduction and decomposition. PhD thesis, PhD thesis, Dept. of ECE, Carnegie Mellon University, 2005.

[29] Zhi Han and Bruce H Krogh. Reachability analysis of large-scale affine systems using lowdimensional polytopes. In International Workshop on Hybrid Systems: Computation and Control, pages 287-301. Springer, 2006.

[30] Colas Le Guernic and Antoine Girard. Reachability analysis of linear systems using support functions. Nonlinear Analysis: Hybrid Systems, 4(2):250-262, 2010.

[31] Mohammad Mahdi Malakiyeh, Saeed Shojaee, and Klaus-Jürgen Bathe. The bathe time integration method revisited for prescribing desired numerical dissipation. Computers 85 Structures, 212:289-298, 2019.

[32] Christopher Rackauckas and Qing Nie. Differentialequations.jl - a performant and feature-rich ecosystem for solving differential equations in julia. The Journal of Open Research Software, 5(1), 2017. Exported from https://app.dimensions.ai on 2019/05/05.

[33] Christian Schilling and Marcelo Forets. JuliaReach/LazySets.jl: v1.45.1. https://github.com/ JuliaReach/LazySets.j1, June 2021. Accessed: 2021-05-31.

[34] S. Schupp, E. Ábrahám, I. Ben Makhlouf, and S. Kowalewski. HyPro: A C++ library for state set representations for hybrid systems reachability analysis. In Proc. NFM'17, volume 10227 of LNCS, pages 288-294. Springer, 2017.

[35] J. K. Scott, D. M. Raimondo, G. R. Marseglia, and R. D. Braatz. Constrained zonotopes: A new tool for set-based estimation and fault detection. Automatica, 69:126-136, 2016.

[36] Thomas Strathmann and Jens Oehlerking. Verifying properties of an electro-mechanical braking system. In ARCH@CPSWeek, volume 34 of EPiC Series in Computing, pages 49-56. EasyChair, 2015.

[37] H.-D. Tran, L. V. Nguyen, and T. T. Johnson. Large-scale linear systems from order-reduction. In Proc. of ARCH16. 3rd International Workshop on Applied Verification for Continuous and Hybrid Systems, page 60-67, 2017. 